\title{
Alpha-Synuclein Pathology in the Submandibular Gland of LRRK2 p.G2019S Mutation Carriers.
}

\section{Dolores Vilas ( $\square$ dvilas.germanstrias@gencat.cat)}

Hospital Universitari Germans Trias i Pujol https://orcid.org/0000-0003-3084-053X

\section{Eduardo Tolosa (DETOLOSA@clinic.cat)}

Hospital Clinic Barcelona

Iban Aldecoa

Hospital Clinic de Barcelona

Joan Berenguer

Hospital Clinic de Barcelona

Isabel Vilaseca

Hospital Clinic de Barcelona

Alex Iranzo

Hospital Clinic de Barcelona

\section{Maria J. Marti}

Hospital Clinic de Barcelona

\section{Carles Martí}

Hospital Clinic de Barcelona

Francisco Lomeña

Hospital Clinic de Barcelona

\section{Llucia Alós}

Hospital Clinic de Barcelona

Ellen Gelpi

Medical University of Vienna

\section{Research}

Keywords: Alpha-synuclein, submandibular gland, LRRK2, G2019S, biomarker, Parkinson

Posted Date: June 12th, 2020

DOI: https://doi.org/10.21203/rs.3.rs-34261/v1

License: (c) (i) This work is licensed under a Creative Commons Attribution 4.0 International License. Read Full License 


\section{Abstract}

Background. The presence of intraneuronal aggregates of phosphorylated alpha-synuclein (pAS), the histological hallmark of Parkinson disease (PD), has been already demonstrated to be present in the autonomic nerve fibres that innervate the submandibular gland in approximately $75 \%$ of living PD patients. The presence of pAS in the peripheral autonomic nervous system in carriers of $L R R K 2$ mutations has not been studied so far. The objective of the current study is to evaluate the presence of abnormal pAS aggregates in the submandibular gland tissue of LRRK2 p.G2019S mutations carriers.

Methods. This is a prospective observational study conducted between 2014 and 2015 at Hospital Clínic de Barcelona, Spain. A random sample of nine asymptomatic LRRK2 (aLRRK2) and 11 LRRK2 associated $\mathrm{PD}$ ( $L R R K 2-\mathrm{PD})$ patients were recruited among a cohort of $\angle R R K 2-\mathrm{PD}$ patients and their relatives already identified at our centre. All the participants underwent transcutaneous needle core biopsy of the submandibular gland under ultrasound guidance. The presence of pAS was assessed in all the participants by immunohistochemistry using anti-Serine 129-phosphorylated AS antibody.

Results. Submandibular biopsy material containing glandular parenchyma was obtained in 4 (44.44\%) aLRRK2 and in 6 (54.55\%) LRRK2-PD patients. Aggregates of pAS were detected in the glandular parenchyma in one of the four (25\%) aLRRK2 subjects and in none ( $0 \%)$ of the $L R R K 2$-PD patients.

Conclusions. Our study shows that pAS aggregates obtained by needle core biopsy of the submandibular gland are infrequent in $L R R K 2$ mutation carriers but may be detected in asymptomatic mutation carriers. The low rate of pAS positive biopsies suggests either a different physiopathology between LRRK2-related and idiopathic PD or that a one-time unilateral submandibular gland biopsy is not the optimal procedure for the study of synuclein aggregation in $L R R K 2$ mutation carriers.

\section{Background}

Abnormal aggregates of phosphorylated alpha-synuclein (pAS) are the major component of the intraneuronal inclusions known as Lewy bodies and Lewy neurites, the histological hallmark of Parkinson disease $(P D)^{1}$. Recently, pAS deposits were detected in the submandibular gland in living patients with idiopathic $\mathrm{PD}^{2,3}$, and also in the prodromal phase of this condition ${ }^{4}$, providing biological evidence of ongoing disease at its earliest stage.

We have assessed the presence of pAS aggregates in the submandibular gland of LRRK2 p.G2019S mutations carriers, both in those with manifest PD (LRRK2-PD) and in asymptomatic carriers (aLRRK2). As $\angle R R K 2$ related CNS neuropathology is not necessarily associated with $\mathrm{pAS}$ aggregates ${ }^{5}$, the detection of pAS in peripheral biopsies in $\angle R R K 2-P D$ cases would identify those with an underlying Lewy type pathology which could be important for implementing potential anti-synuclein therapies. In a $L R R K 2$, the presence of peripheral pAS could reflect an underlying synucleinopathy and constitute a risk marker for the later development of manifest PD. 


\section{Methods}

This is a prospective observational study conducted between July 2014 and May 2015 at the Hospital Clinic de Barcelona, Spain. The ethical committee at our institution approved the study and all participants gave their written informed consent.

\section{Participants}

At our institution five groups of subjects underwent biopsy of the submandibular gland for research: a LRRK2, LRRK2-PD patients, idiopathic (iPD), idiopathic REM sleep behaviour disorder (IRBD) and controls. Results from the IRBD, iPD patients and controls were previously reported ${ }^{4}$. Herein we present the results from the a LRRK2 and $L R R K 2-\mathrm{PD}$ participants.

Asymptomatic LRRK2 mutation carriers and LRRK2-PD patients. Nine a LRRK2 carriers of the p.G2019S mutation and eleven LRRK2-PD patients were selected among a cohort of $L R R K 2$-PD patients and their relatives. Participants had been already identified at our centre, had been screened for LRRK2 mutations as previously described ${ }^{6}$, and were positive for the LRRK2 p.G2019S mutation. Participation in the study was proposed to all who were alive and accessible. PD was diagnosed according to United Kingdom PD Society criteria with the exception that a positive family history was not considered an exclusion criterion ${ }^{7}$.

Exclusion criteria in all participants were current or past medical history of disorders affecting the salivary glands (e.g., chronic sialadenitis, abscesses, active neoplasms, Sjögren syndrome), coagulation disorders, and anticoagulant or antiplatelet drug intake.

\section{Clinical assessments}

Demographic and clinical data were collected. Parkinsonism was assessed by the motor part of the Movement Disorders Society Unified Parkinson's Disease Rating Scale, ${ }^{8}$ the Non-motor symptoms questionnaire (NMSQ) ${ }^{9}$ was applied, smell function was assessed by the Spanish-version of the 40 -item University of Pennsylvania Smell Identification Test (UPSIT), ${ }^{10}$ constipation by Rome III Diagnostic Criteria, ${ }^{11}$ depression by DSM-IV criteria, ${ }^{12}$ RBD by clinical and video-polysomnographic established criteria $^{13}$ or suspected by the RBD screening questionnaire (RBDSQ) ${ }^{14}$ and a clinical interview. Echogenicity of the substantia nigra was assessed by means of transcranial sonography as previously described. ${ }^{15}$ Dopamine transporter imaging with ${ }^{123} \mathrm{l}-2 \beta$-carbomethoxy-3 $\beta$-(4-iodophenyl)-N-(3-fluoropropyl)-nortropane single photon emission computed tomography (DaT-SPECT) was performed as previously described. ${ }^{16}$

\section{Procedures}

Submandibular gland biopsy and alpha-synuclein immunohistochemistry of the submandibular gland were assessed as described previously ${ }^{4}$. Briefly, a core needle biopsy was performed in all participants 
using a commercial 16-gauge needle. Biopsies were performed unilaterally under ultrasound guidance after subcutaneous infiltration of local anesthesia. Between 2-4 punctures were performed by an experienced radiologist who was masked to the clinical status of the participants. After each procedure, specimens were immediately processed and serial $4 \mu \mathrm{m}$ sectioning of the whole specimens was performed. Three slides were selected from the first, middle or final third of the blank slide set from each subject and were stained with haematoxylin-eosin. Immunohistochemistry was performed on the third set with most submandibular parenchyma and/or nervous s tissue using an automated Dako Autostainer using antiSerine 129-phosphorylated alpha-synuclein antibody (Wako clone pSyn\#64; Pure Chemical Industries, Osaka, Japan)), as described previously ${ }^{4}$. pAS immunoreactivity in nervous structures within or outside the submandibular gland parenchyma was assessed as present or absent. At the time of the histopathologist examination, neuropathologists were blinded regarding the clinical condition of the individual's tissues.

\section{Results}

Demographic and clinical data of participants are summarized in Tables 1 and 2. Biopsy specimens contained submandibular gland parenchyma in 6 of the 11 (54.55\%) LRRK2-PD patients and in 4 of the 9 (44.44\%) a LRRK2. The remaining samples contained periglandular connective tissue with variable amount of vessels and nerve fibres or muscle. 
Table 1

Demographic and clinical data.

\begin{tabular}{|c|c|c|}
\hline & $\begin{array}{l}\mathrm{a} L R R K 2 \\
(\mathrm{n}=9)\end{array}$ & $\begin{array}{l}\text { LRRK2PD } \\
(\mathrm{n}=11)\end{array}$ \\
\hline Age (years) & $48.89 \pm 8.43$ & $57.0 \pm 13.0$ \\
\hline Male, n (\%) & $5(55.56)$ & $5(45.5)$ \\
\hline PD duration (years) & - & $4.30 \pm 4.14$ \\
\hline NMSQ score & $5.33 \pm 5.27$ & $23.70 \pm 33.26$ \\
\hline MDS-UPDRS I & $5.00 \pm 3.77$ & $8.91 \pm 7.70$ \\
\hline MDS-UPDRS II & $1.67 \pm 3.94$ & $11.18 \pm 5.96$ \\
\hline MDS- UPDRS III & $0.78 \pm 1.39$ & $20.82 \pm 7.43$ \\
\hline Hoehn and Yahr & - & $1.91 \pm 0.54$ \\
\hline UPSIT score & $33.56 \pm 2.19$ & $23.30 \pm 5.74$ \\
\hline RBDSQ score & $1.67 \pm 2.60$ & $1.09 \pm 1.92$ \\
\hline \multicolumn{3}{|c|}{$\begin{array}{l}\text { Values are reported as media, standard deviation, number and percentage. aLRRK2: asymptomatic } \\
\text { carriers of the p.G2019S LRRK2 mutation. LRRK2-PD: LRRK2 associated Parkinson's disease. IPD: } \\
\text { Idiopathic Parkinson's disease. PD: Parkinson's disease. NMSQ: Non-motor symptoms questionnaire. } \\
\text { MDS-UPDRS I, II and III: Movement Disorders Society Unified Parkinson's Disease Rating Scale parts I, II } \\
\text { and III. UPSIT: University of Pennsylvania Smell Identification Test. RBDSQ: RBD screening } \\
\text { questionnaire. }\end{array}$} \\
\hline
\end{tabular}


Table 2

Demographic, clinical and imaging characteristics of the asymptomatic LRRK2 carriers.

\begin{tabular}{|c|c|c|c|c|c|c|c|c|c|}
\hline Patients & 1 & 2 & 3 & 4 & 5 & 6 & 7 & 8 & 9 \\
\hline $\begin{array}{l}\text { Presence of } \\
\text { glandular } \\
\text { parenchyma }\end{array}$ & Yes & Yes & Yes & Yes & No & No & No & No & No \\
\hline pAS+ & Yes & No & No & No & No & No & No & No & No \\
\hline Sex & Female & Female & Male & Male & Male & Male & Male & Female & Female \\
\hline $\begin{array}{l}\text { Age at biopsy } \\
\text { (years) }\end{array}$ & 60 & 45 & 48 & 39 & 64 & 48 & 47 & 50 & 39 \\
\hline Depression & No & No & No & No & No & No & No & No & No \\
\hline Constipation & No & No & No & No & No & No & No & No & No \\
\hline RBDSQ score & 0 & 0 & 7 & 2 & 1 & 5 & 0 & 0 & 0 \\
\hline UPSIT-40 score & 31 & 32 & 37 & 35 & 30 & 35 & 34 & 34 & 34 \\
\hline Hyposmia & No & No & No & No & No & No & No & No & No \\
\hline NMSQ score & 14 & 4 & 3 & 0 & 0 & 12 & 0 & 7 & 8 \\
\hline $\begin{array}{l}\text { MDS-UPDRS-I } \\
\text { score }\end{array}$ & 5 & 4 & 8 & 1 & 4 & 3 & 0 & 8 & 12 \\
\hline $\begin{array}{l}\text { MDS-UPDRS II } \\
\text { score }\end{array}$ & 0 & 0 & 1 & 0 & 2 & 0 & 0 & 0 & 12 \\
\hline $\begin{array}{l}\text { MDS-UPDRS III } \\
\text { score }\end{array}$ & 0 & 0 & 0 & 0 & 0 & 2 & 0 & 1 & 4 \\
\hline MOCA & 30 & 30 & 27 & 30 & 29 & 23 & 30 & 20 & 26 \\
\hline $\begin{array}{l}\text { Substantia nigra } \\
\text { hyperechogenicity }\end{array}$ & No & No & No & No & Yes & Yes & No & Yes & No \\
\hline $\begin{array}{l}\text { Reduced striatal } \\
\text { uptake in } \\
\text { dopamine } \\
\text { transporter } \\
\text { imaging }\end{array}$ & No & No & No & No & No & No & $\begin{array}{l}\text { Not } \\
\text { done }\end{array}$ & No & $\begin{array}{l}\text { Not } \\
\text { done }\end{array}$ \\
\hline \multicolumn{10}{|c|}{$\begin{array}{l}\text { RBD: REM sleep behavior disorder; RBDSQ: RBD screening questionnaire; UPSIT: University of } \\
\text { Pennsylvania Smell Identification Test; NMSQ: non-motor symptoms questionnaire; MDS-UPDRS: } \\
\text { Movement Disorders Society Unified Parkinson Disease Rating Scale, parts I, II and III; MOCA: Montreal } \\
\text { Cognitive Assessment. }\end{array}$} \\
\hline
\end{tabular}

Aggregates of pAS were detected in the glandular parenchyma in one of the four (25\%) aLRRK2 subjects (Fig. 1) and in none (0\%) of the six $\angle R R K 2$-PD patients with available glandular tissue. pAS aggregates in the a $L R R K 2$ individual were identified in nerve structures of the connective tissue within the gland, rarely 
surrounding individual glands. This type of aggregates has been shown to co-distribute with tyrosinehydroxylase positive sympatethic nerve fibres ${ }^{4}$.

None of the aLRRK2 or the LRRK2-PD showed extraglandular pAS aggregates.

\section{Asymptomatic LRRK2 p.G2019S carriers (Tables 1 and 2)}

The mean age of the a $L R R K 2$ was $48.89 \pm 8.43$ years. The mean UPSIT score was $33.56 \pm 2.19$ points, and none of the nine a $L R R K 2$ had hyposmia. None of the a $L R R K 2$ had depression, constipation or RBD by the RBSQ. Seven of the nine aLRRK2 underwent a DaT-SPECT, which was normal in all of them. Three out of seven (42.9\%) individuals who underwent transcranial sonography had hyperechogenicity of the substantia nigra .

The asymptomatic LRRK2 mutation carrier who showed pAS pathology in the submandibular glandular parenchyma was a 60-year-old woman. Her score in the NMSQ was 14, with all points got from the sleep subscore due to insomnia. Her neurological examination was normal, without evidence of parkinsonian signs. A conducted interview detected no history of dream-enacting behaviours, constipation or depression. She had no hyposmia. The echogenicity of the substantia nigra and the DaT-SPECT were normal. At the age of 65 , she is still asymptomatic.

\section{Discussion}

To the best of our knowledge this is the first study that assessed the presence of pAS pathology in the submandibular gland of $L R R K 2$ mutation carriers. pAS aggregates were found in one of the four a $L R R K 2$ in whom glandular parenchyma was available after transcutaneous needle core biopsy. None of the LRRK2PD patients had pAS aggregates in the submandibular gland or extraglandular tissues. Submandibular gland parenchyma was obtained only in around $50 \%$ of the $\angle R R K 2$-PD and a $L R R K 2$ individuals.

The absence of pAS positivity in manifest LRRK2-PD was not expected, since pAS accumulation in the peripheral autonomic nervous system is thought to reflect an ongoing synucleinopathy, which occurs in the majority of, but not all, LRRK2-G2019S-PD cases. Still, in a study of alpha-synuclein aggregation in cerebrospinal fluid by real-time quaking-induced conversion (RT-QuIC) ${ }^{17}$ the percentage of positive cases in LRRK2-PD was also much lower than in iPD (40\% vs $90 \%)$. The pleiomorphic pathology of LRRK2-PD linked to the p.G2019S mutation may in part explain the results. Other possible explanations include the possibility that, unlike in iPD, peripheral autonomic nervous system Lewy type pathology is less prominent or even absent in p.G2019S LRRK2-PD patients. This could be supported by the notion that dysautonomia seems to occur less frequently in p.G2019S $\angle R R K 2-\mathrm{PD}$ than in $\mathrm{iPD}^{18}$, although no definitive data are available. Also possible is that synuclein pathology might have been present in the peripheral autonomic system at an early disease stage and migrated later centripetally to the central nervous system, becoming undetectable in manifest $\angle R R K 2-\mathrm{PD}$, as has been speculated for idiopathic $\mathrm{PD}^{19}$. Finally, a sampling bias with under-representative tissue samples cannot be excluded. 
The number of submandibular glands with pAS positivity among a LRRK2 was low (25\%). Of interest, the percentage of positive cases is close to the proportion of a $L R R K 2$ reported to have misfolded synuclein in the cerebrospinal fluid (18.8\%) assessed by RT-QuIC ${ }^{17}$. Factors such the relatively young age of the patients may also influence these results since parkinsonism appears generally late in LRRK2-PD. However, even if our patients possibly destined to develop PD might have been disease free at the time of the biopsy, manifest $L R R K 2 P D$ were also pAS negative.

The main limitations of our study are the small sample size which precludes generalization of the results and the low frequency of glandular tissue obtained with unilateral transcutaneous needle biopsy of the submandibular gland despite the use of ultrasound guiadance. In previous studies in living PD using a similar biopsy methodology but without ultrasound guidance, submandibular glandular tissue was not obtained in $20-24 \%$ of participants ${ }^{2-3}$. Bilateral transcutaneous needle biopsies of the submandibular gland in PD patients seemed recently feasible and safe, showing a better tissue acquisition ${ }^{21}$. Technical refinement of the procedure is needed to improve the ability to obtain glandular parenchyma in living subjects.

In conclusion, our study shows that pAS aggregates obtained by needle core biopsy of the submandibular gland may be detected in a LRRK2, suggesting that Lewy type pathology is already ongoing in a subset of subjects. However, the low rate of submandibular gland tissue obtained by the biopsies and the fact that none of the LRRK2-PD patients showed pAS aggregates suggest that a one-time unilateral submandibular gland biopsy may not be the optimal procedure for the study of pAS in LRRK2 p.G2019S mutation carriers. Other peripheral tissues such as skin or minor salivary glands that are easily accessible may prove a better target for tissue-based studies of synuclein aggregates in LRRK2 disease. Yet emerging synuclein amplification methods in cerebrospinal fluid ${ }^{16}$ or in peripheral tissues including the submandibular gland ${ }^{20}$ may turn out to be better markers of central and/or peripheral synuclein aggregation in LRRK2-PD.

\section{Abbreviations}

aLRRK2: asymptomatic $L R R K 2$ carriers

iPD: idiopathic Parkinson disease

DaT-SPECT: $123 \mathrm{I}-2 \beta$-carbomethoxy-3 $\beta$-(4-iodophenyl)-N-(3-fluoropropyl)-nortropane single photon emission computed tomography

IRBD: idiopathic REM sleep behaviour disorder

LRRK2-PD: LRRK2 associated Parkinson disease

NMSQ: Non-motor symptoms questionnaire

pAS: phosphorylated alpha-synuclein 
UPSIT: University of Pennsylvania Smell Identification Test

\section{Declarations}

\section{Ethics approval and consent to participate:}

The ethical committee at Hospital Clínic de Barcelona approved the study and all participants gave their written informed consent.

\section{Consent for publication:}

Not applicable.

\section{Availability of data and materials:}

The datasets generated and/or analysed during the current study are available from the corresponding author on reasonable request.

\section{Competing interests:}

Eduardo Tolosa received honoraria in the past 12 months for consultancy from Zambon, TEVA, Bial, Boehringer Ingelheim,Denali, Prevail, Abbie and BIOGEN and has received funding for research from Spanish Network for Research on Neurodegenerative Disorders (CIBERNED)- Instituto de Salud Carlos III (ISCIII), and The Michael J. Fox Foundation for Parkinson's Research(MJFF). Dolores Vilas, Iban Aldecoa, Joan Berenguer, Isabel Vilaseca, Alex Iranzo, Maria J. Marti, Carles Martí, Francisco Lomeña, Llucia Alós and Ellen Gelpi declare no competing interests.

\section{Funding:}

This study had not funding.

\section{Authors' contributions:}

DV and ET designed the study, analyzed and interpreted the patient data and wrote the manuscript. IA, LA and EG performed the histological examination of the biopsy samples. JB, CM and IV performed the submandibular gland biopsies. Al and MJM analyzed and interpreted the patient data. FL interpreted the DAT-SPECT imaging. All authors read and approved the final manuscript. 


\section{Acknowledgements:}

The authors are grateful to the patients and the families that participated in the study.

\section{References}

1. Dickson DW, Braak H, Duda JE, Duyckaerts C, Gasser T, Halliday, et al. Neuropathological assessment of Parkinson's disease: refining the diagnostic criteria. Lancet Neurol. 2009;8:1150-1157.

2. Adler CH, Dugger BN, Hinni ML, Lott DG, Driver-Dunckley E, Hidalgo J, et al. Submandibular gland needle biopsy for the diagnosis of Parkinson disease. Neurology. 2014;82:858-864.

3. Adler CH, Dugger BN, Hentz JG, Hinni ML, Lott DG, Driver-Dunckley E, et al. Peripheral synucleinopathy in early Parkinson's disease: submandibular gland needle biopsy findings. Mov Disord. 2017;32:722723.

4. Vilas D, Iranzo A, Tolosa E, Aldecoa I, Berenguer J, Vilaseca I, et al. Assessment of a-synuclein in submandibular glands of patients with idiopathic rapid-eye-movement sleep behaviour disorder: a case-control study. Lancet Neurol. 2016:708-718.

5. Gaig C, Martí MJ, Ezquerra M, Cardozo A, Rey MJ, Tolosa E.. G2019S LRRK2 mutation causing Parkinson's disease without Lewy bodies. BMJ Case Rep. 2009;2009. doi: 10.1136/bcr.08.2008.0632.

6. Gaig C, Ezquerra M, Marti MJ, Muñoz E, Valldeoriola F, Tolosa E. LRRK2 mutations in Spanish patients with Parkinson disease: frequency, clinical features, and incomplete penetrance. Arch Neurol. 2006;63:377-82.

7. Hughes AJ, Daniel SE, Kilford L, Lees AJ. Accuracy of clinical diagnosis of idiopathic Parkinson's disease: a clinico-pathological study of 100 cases. J Neurol Neurosurg Psychiatry. 1992;55:181-4.

8. Goetz CG, Tilley BC, Shaftman SR, Stebbins GT, Fahn S, Martinez-Martin P, et al. Movement Disorder Society-sponsored revision of the Unified Parkinson's Disease Rating Scale (MDS-UPDRS): scale presentation and clinimetric testing results. Mov Disord. 2008;23:2129-70.

9. Chaudhuri KR, Martinez-Martin P, Schapira AH, Stocchi F, Sethi K, Odin P, et al. International multicenter pilot study of the first comprehensive self-completed nonmotor symptoms questionnaire for Parkinson's disease: The NMS Quest Study. Mov Disord 2006;21:916-923.

10. Doty RL. The Smell Identification TestTM administration manual. Philadephia: Sensonics, Inc., Third ed., 1995.

11. Longstreth GF, Thompson WG, Chey WD, Houghton LA, Mearin F, Spiller RC. Functional bowel disorders. Gas- troenterology. 2006;130:1480-1491.

12. Diagnostic and Statistical Manual of Mental Disorders: DSM-IV, American Psychiatric Association, Washington, 1994

13. American Academy of Sleep Medicine. International classification of sleep disorders, 3rd ed. Darien, IL: American Academy of Sleep Medicine, 2014.

14. Stiasny-Kolster K, Mayer G, Schäfer S, Möller JC, Heinzel-Gutenbrunner M, Oertel WH. The REM sleep behavior disorder screening questionnaireea new diagnostic instrument. Mov. Disord. 2007:2386- 
2393.

15. Vilas D, Iranzo A, Pont-Sunyer C, Serradell M, Gaig C, Santamaria J, Tolosa E. Brainstem raphe and substantia nigra echogenicity in idiopathic REM sleep behavior disorder with comorbid depression. J Neurol. 2015;262:1665-1672.

16. Iranzo A, Lomeña F, Stockner H, Valldeoriola F, Vilaseca I, Salamero M, et al. Decreased striatal dopamine transporter uptake and substantia nigra hyperechogenicity as risk markers of synucleinopathy in patients with idiopathic rapid-eye-movement sleep behaviour disorder: a prospective study. Lancet Neurol. 2010;9:1070-1077.

17. Garrido A, Fairfoul G, Tolosa E, Martí MJ, Green A. a-synuclein RT-QulC in cerebrospinal fluid of LRRK2linked Parkinson's disease. Ann Clin Transl Neurol. 2019;6:1024-1032.

18. Valldeoriola F, Gaig C, Muxi A, Navales I, Paredes P, Lomeña F, et al. 123I-MIBG cardiac uptake and smell identification in parkinsonian patients with LRRK2 mutations. J Neurol. 2011;258:1126-32.

19. Orimo S, Uchihara T, Nakamura A, Mori F, Kakita A, Wakabayashi K, et al. Axonal alpha-synuclein aggregates herald centripetal degeneration of cardiac sympathetic nerve in Parkinson's disease. Brain 2008;131:642-650.

20. Manne S, Kondru N, Jin H, Anantharam V, Huang X, Kanthasamy A, et al. a-Synuclein real-time quaking-induced conversion in the submandibular glands of Parkinson's disease patients. Mov Disord. 2020;35(2):268-278.

21. Adler CH, Serrano GE, Zhang N, Hinni ML, Lott DG, Mehta SH, et al. Feasibility of repeat and bilateral submandibular gland needle biopsies in Parkinson's disease. Parkinsonism Relat Disord. 2019;68:6972.

\section{Figures}
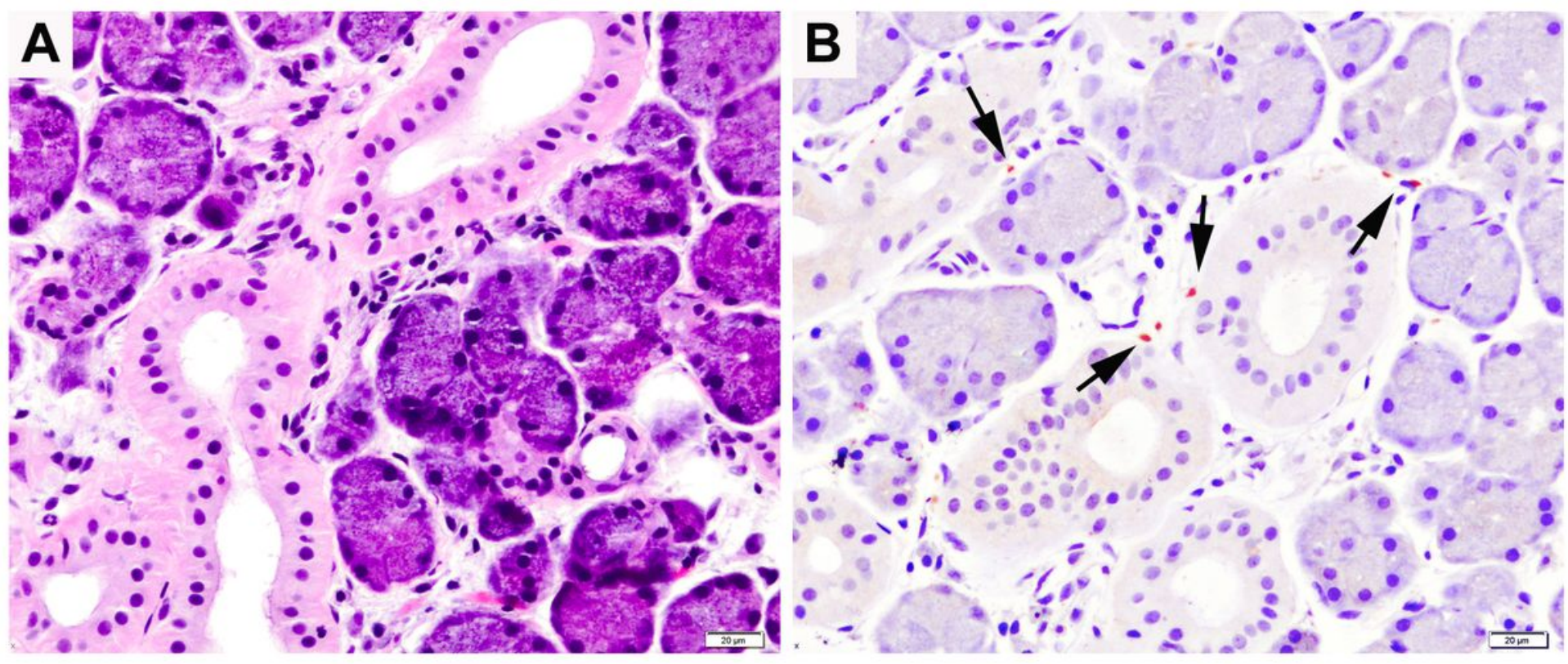

Figure 1 
A: Representative histological image of the submandibular biopsy (haematoxylin-eosin stain). B: Immunohistochemistry for phosphorylated alpha-synuclein shows small aggregates surrounding glandular structures (brown signal, arrows) in areas corresponding to the course of autonomic nerve fibres. Scale bars: $20 \mu \mathrm{m}$. 